\title{
Associations between body mass index and the risk of renal events in patients with type 2 diabetes
}

Kamel Mohammedi ${ }^{1,2,3}$, John Chalmers ${ }^{1}$, William Herrington ${ }^{4}$, Qiang Lii ${ }^{1}$ Giuseppe Mancia ${ }^{5}$, Michel Marre ${ }^{2,3,6}$, Neil Poulter ${ }^{7}$, Anthony Rodgers ${ }^{1}$, Bryan Williams ${ }^{8}$, Vlado Perkovic ${ }^{1}$, Josef Coresh ${ }^{9}$ and Mark Woodward ${ }^{1,9,10}$

\begin{abstract}
Background/objectives: We aimed to evaluate the relationship between BMI and the risk of renal disease in patients with type 2 diabetes in the Action in Diabetes and Vascular Disease: PreterAx and DiamicroN Modified-Release Controlled Evaluation (ADVANCE) study.

Subjects/methods: Participants were divided into six baseline BMI categories: $<18.5$ (underweight, $n=58$ ); $\geq 18.5$ to $<25$ (normal, $n=2894$ ); $\geq 25$ to $<30$ (overweight, $n=4340$ ); $\geq 30$ to $<35$ (obesity grade $1, n=2265$ ); $\geq 35$ to $<40$ (obesity grade $2, n=744$ ); and $\geq 40 \mathrm{~kg} / \mathrm{m}^{2}$ (obesity grade $3, n=294$ ); those underweight were excluded. The composite outcome "major renal event" was defined as development of new macroalbuminuria, doubling of creatinine, end stage renal disease, or renal death. These outcomes and development of new microalbuminuria were considered individually as secondary endpoints.

Results: During 5-years of follow-up, major renal events occurred in 487 (4.6\%) patients. The risk increased with higher BMI. Multivariable-adjusted HRs (95\% Cls), compared to normal weight, were: $0.91(0.72-1.15)$ for overweight; 1.03 $(0.77-1.37)$ for obesity grade $1 ; 1.42(0.98-2.07)$ for grade 2 ; and $2.16(1.34-3.48)$ for grade 3 ( $p$ for trend $=0.006)$. These findings were similar across subgroups by randomised interventions (intensive versus standard glucose control and perindopril-indapamide versus placebo). Every additional unit of BMI over $25 \mathrm{~kg} / \mathrm{m}^{2}$ increased the risk of major renal events by $4(1-6) \%$. Comparable results were observed with the risk of secondary endpoints.
\end{abstract}

Conclusions: Higher BMI is an independent predictor of major renal events in patients with type 2 diabetes. Our findings encourage weight loss to improve nephroprotection in these patients.

\section{Introduction}

Globally, obesity is common with alarming rates of increasing prevalence ${ }^{1,2}$. It is a key component of the metabolic syndrome, which is also characterised by hypertension, dyslipidaemia, and insulin resistance, and often leads to type 2 diabetes $^{3}$. Diabetes is a leading cause of chronic kidney disease (CKD) and end-stage renal disease $(E S R D)^{4}$. In the absence of diabetes,

\footnotetext{
Correspondence: John Chalmers (chalmers@georgeinstitute.org.au)

${ }^{1}$ The George Institute for Global Health, University of Sydney, Sydney, Australia ${ }^{2}$ INSERM, UMRS 1138, Centre de Recherche des Cordeliers, Paris, France Full list of author information is available at the end of the article
}

however, experimental and epidemiological studies have also provided accumulating evidence that obesity is an independent risk factor for $\mathrm{CKD}^{5,6}$, a risk mediated in part through intraglomerular hypertension and hyperfiltration ${ }^{7}$.

Most reported observational studies have found positive associations between being overweight or obese and kidney outcomes (which include development of CKD, rapid changes in kidney function or ESRD) ${ }^{8-12}$. However, few have been large enough to compare people with and without diabetes reliably ${ }^{9}$, and there remains some uncertainty in people with diabetes as to whether higher 
body mass index (BMI) increases risk of developing macroalbuminuria, and whether BMI-CKD associations are mediated through differences in renal risk factors affected by adiposity (e.g., glycemia and blood pressure).

In the present study, we aimed to evaluate the relationship between baseline BMI and major renal events among patients with type 2 diabetes in the Action in Diabetes and Vascular Disease: PreterAx and DiamicroN Modified-Release Controlled Evaluation (ADVANCE) trial (ClinicalTrials.gov number, NCT00145925). In order to investigate potential mechanisms for any associations, outcomes were analysed for the whole cohort and in subgroups for those randomised to different intensities of long-term glycaemic control and to use of placebo versus perindopril-indapamide, an ACE-inhibitor/diuretic combination drug that would be expected to reduce glomerular hyperfiltration.

\section{Materials/subjects and methods Study population}

The ADVANCE study was a $2 \times 2$ factorial randomised controlled trial which tested the effects of intensive glucose control using a gliclazide-MR-based regimen, and routine blood pressure treatment using a fixed-dose combination of perindopril and indapamide, on the incidence of major macrovascular and microvascular events in patients with type 2 diabetes. The design and clinical characteristics of participants have been published previously ${ }^{13-15}$. Briefly, patients aged 55 years or older with diabetes diagnosed at 30 years or older with pre-existing cardiovascular disease or with at least one risk factor for cardiovascular disease were eligible. Participants were followed prospectively for clinical events and had blood pressure and urinary albumin to creatinine ratio (ACR) measured at local study clinics at 2-year, 4-year and final follow-up visits. The ADVANCE protocol was approved by the Institutional Ethics Committee of each participating centre and all participants provided written informed consent before their enrolment in the trial.

\section{Definition of BMI categories at baseline}

Baseline BMI, computed as the weight in kilograms divided by the square of the height in metres, was categorised at baseline into six categories according to the World Health Organization classification ${ }^{16}$ : underweight $(<18.5)$, normal weight $(\geq 18.5$ to $<25)$, overweight ( $\geq 25$ to $<30$ ), and obesity grade $1(\geq 30$ to $<35$ ), grade 2 ( $\geq 35$ to $<40)$, and grade $3\left(\geq 40 \mathrm{~kg} / \mathrm{m}^{2}\right)$.

\section{Primary and secondary endpoints}

As pre-specified in the ADVANCE protocol ${ }^{13}$, "major renal events" were defined as a composite of macroalbuminuria (defined as a urinary ACR $>300 \mathrm{mg} / \mathrm{g}$ ), doubling of the serum creatinine level to at least $200 \mu \mathrm{mol} / \mathrm{l}$, ESRD (defined as the need for renal-replacement therapy), or death due to renal disease. "New cases of microalbuminuria" (defined as $30<\mathrm{ACR} \leq 300 \mathrm{mg} / \mathrm{g}$ ), "Development of new macroalbuminuria", and "doubling of creatinine, ESRD, or renal death", were considered individually as secondary endpoints. The primary endpoints were reviewed by an independent End Point Adjudication Committee.

\section{Statistical analyses}

Clinical and biological characteristics of participants at baseline were presented both overall and according to BMI categories. Categorical variables were expressed as the number of patients with the corresponding percentage, and continuous variables as mean (SD), or as median (interquartile interval) for those with a skewed distribution. Patients with missing data regarding estimated glomerular filtration rate (eGFR) and ACR at baseline $(n=545)$ were excluded from the current study. Few $(n=58 ; 0.6 \%)$ patients were underweight, and so these were also excluded from the main set of analyses, although included in a sensitivity analysis. Cox proportional hazards regression models were fitted to estimate hazard ratios (HRs), with associated 95\% confidence intervals (CI), for major renal events by BMI categories, taking normal weight as the reference group. The primary model (model 1) adjusted for baseline age, sex, region of origin (Asia: Philippines, China, Malaysia, and India; established market economies: Australia, Canada, France, Germany, Ireland, Italy, Netherlands, New Zealand, United Kingdom; and Eastern Europe: Czech Republic, Estonia, Hungary, Lithuania, Poland, Russia, Slovakia), prior cardiovascular disease (defined as the presence at baseline of myocardial infarction, stroke, coronary artery bypass graft, percutaneous transluminal coronary angioplasty, hospital admission for unstable angina or transient ischaemic attack), eGFR (computed by the CKD-Epidemiology Collaboration equation) $)^{17}$, squared eGFR, urinary ACR, history of ever smoking and study allocation. In model 2 we additionally adjusted for baseline duration of diabetes, HbA1c, systolic blood pressure, total-cholestrol and HDL-cholesterol, and triglycerides. Since these are factors that BMI can be expected to affect causally, most results presented are from model 1 . We also evaluated the association of BMI as a continuous variable with major renal events using piece-wise linear splines with knots at $18.5,25,30,35,40$, and $45 \mathrm{~kg} / \mathrm{m}^{2}$, and a reference value at $21 \mathrm{~kg} / \mathrm{m}^{2}$. The hazard ratio for major renal events associated with each single additional unit of BMI above $25 \mathrm{~kg} / \mathrm{m}^{2}$ was also estimated.

Sensitivity analyses were performed to test the association of BMI categories with the risk of major renal events: (i) in different groups of randomised study treatment (standard and intensive glucose control; placebo and perindopril-indapamide) considered separately; (ii) in different CKD stages (stage 1 [eGFR $\geq 90 \mathrm{~mL} / \mathrm{min} / 1.73$ 
$\mathrm{m}^{2}$ ]; stage $2[\geq 60$ to $<90]$, and stage $3[<60]$ ); (iii) after treating non-renal death as a competing risk using the Fine and Gray method ${ }^{18}$; (iv) in participants who did not change their BMI category during follow-up; (v) the association of BMI categories with the risk of new microalbuminuria in patients with normoalbuminuria at baseline; and (vi) after including patients with underweight, who were otherwise omitted.

Statistical analyses were performed using SAS software, version 9.3 (SAS Institute, www.sas.com) and Stata software version 13 (StataCorp., www.stata.com). A $p$-value $<0.05$ was considered significant.

\section{Results}

\section{Baseline characteristics by BMI categories}

Among 10,537 participants investigated, 58\% were men, and 38, 43, and 19\% were from Asia, Established market economies, and Eastern Europe, respectively (Table 1). Their mean (SD) age and duration of diabetes were 66(6) and $8(6)$ years, respectively, and their mean HbA1c was $7.5(1.5) \%$. Mean (SD) BMI at baseline was $28(5) \mathrm{kg} / \mathrm{m}^{2}$, and 2894 (27\%), 4340 (41\%), 2265 (22\%), 744 (7\%) and 294 (3\%) patients, respectively, were in the normal weight, overweight and obesity grades 1, 2 and 3 categories. Mean eGFR was $75(17) \mathrm{mL} / \mathrm{min} / 1.73 \mathrm{~m}^{2}$, and 2341 (22\%), 5952 (57\%) and 2244 (21\%) were categorised as CKD stages 1, 2 , and 3, respectively. Median urinary ACR was 15(7-40) $\mathrm{mg} / \mathrm{g}$, with 7312 (69\%), $2824(27 \%)$ and $401(4 \%)$ in the normo-albuminuric, micro-albuminuric and macroalbuminuric ranges.

Compared to those with normal weight, patients with obesity were more frequently from established market economies, had a shorter duration of diabetes, and greater systolic blood pressure, and serum triglycerides concentration. They were more likely to use antihypertensive and lipid lowering treatments, and to have ever smoked.

\section{Risk of major renal events during follow-up by BMI categories}

Major renal events occurred in 487 (4.6\%) participants during a median duration of follow-up of 5.0 (interquartile interval: 4.5-5.0) years. Patients who developed major renal events during follow-up, compared to those who did not, were more frequently men, had a longer duration of diabetes at baseline, higher systolic blood pressure, HbA1c, and urinary ACR levels, had a lower eGFR, and were more likely to use antihypertensive and lipid lowering drugs (Supplemental Table S1). Major renal events occurred in 144 (5.0\%), 181 (4.2\%), 96 (4.2\%), 43 (5.8\%), and $23(7.8 \%)$ participants with normal weight, overweight, and obesity grades 1, 2 and 3, respectively (Table 2). The risk of major renal events increased gradually across increasing BMI categories, and the highest risk was observed in patients with severe obesity. Adjusted HRs (95\% CIs) from model 1, compared to normal weight, were: overweight: $0.91(0.72-1.15)$, obesity grade $1: 1.03$ (0.77-1.37), grade 2: $1.42(0.98-2.07)$, and grade 3: 2.16 $(1.34-3.48, p$ for trend $=0.006)$. Very similar results were observed when additional adjustments, including mediating factors, were included (model 2) - as was the case for the remaining analyses (results not shown). The same pattern was seen when BMI was fitted as a continuous variable (Fig. 1). Above $25 \mathrm{~kg} / \mathrm{m}^{2}$, the association of BMI with major renal events appeared to be log-linear, and each additional unit was associated with $4(1-6) \%$ increased risk $(p=0.002)$.

\section{Risk of secondary endpoints during follow-up according to BMI categories at baseline}

New cases of microalbuminuria, macroalbuminuria, and doubling of creatinine, ESRD or renal death occurred during follow-up in 2730 (25.9\%), 389 (3.5\%) and 162 (1.5\%) participants, respectively. The risk of new microalbuminuria or macroalbuminuria increased gradually across increasing BMI categories (Table 3). The risk of doubling of creatinine, ESRD or renal death seems to be higher in patients with obesity stages 2 and 3, but the test for trend was non-significant. Each additional unit of BMI over $25 \mathrm{~kg} / \mathrm{m}^{2}$ increased the risk of microalbuminuria $(p=0.0008)$, macroalbuminuria $(p=0.004)$, and doubling of creatinine, ESRD or renal death $(p=$ 0.008 ) by $2(1-3), 4(1-6)$, and $5(1-10) \%$, respectively (using model 1).

\section{Sensitivity analyses}

The associations of BMI categories with the risk of major renal events were compared in different groups of study treatments (Table 4, $p$ for interaction between trend in BMI and glucose lowering control $=0.14$ and $p$ for interaction between trend in BMI and blood pressure treatment $=0.96$ ), as well as in different baseline CKD stages $(p$ for interaction $=0.14$, Supplemental Table S2) and remained significant after treating non-renal death as a competing risk ( $p$ for trend $=0.01$, Supplemental Table S3). During follow-up, 7103 (67\%) participants maintained the same BMI categories as at baseline (Supplemental Table S4). When we considered only these participants, BMI categories remained significantly associated with major renal events ( $p$ for trend $=0.002$, Supplemental Table S5). Similarly, the association of BMI categories with increasing risk of new microalbuminuria remained significant ( $p$ for trend $=0.02$ ) in patients with normoalbuminuria at baseline (Supplemental Table S6). Finally, when we considered the entire cohort, underweight was associated with a higher risk of major renal events compared to normal weight (HR 2.17, 95\% CI 1.01-4.67) (using model 1). 


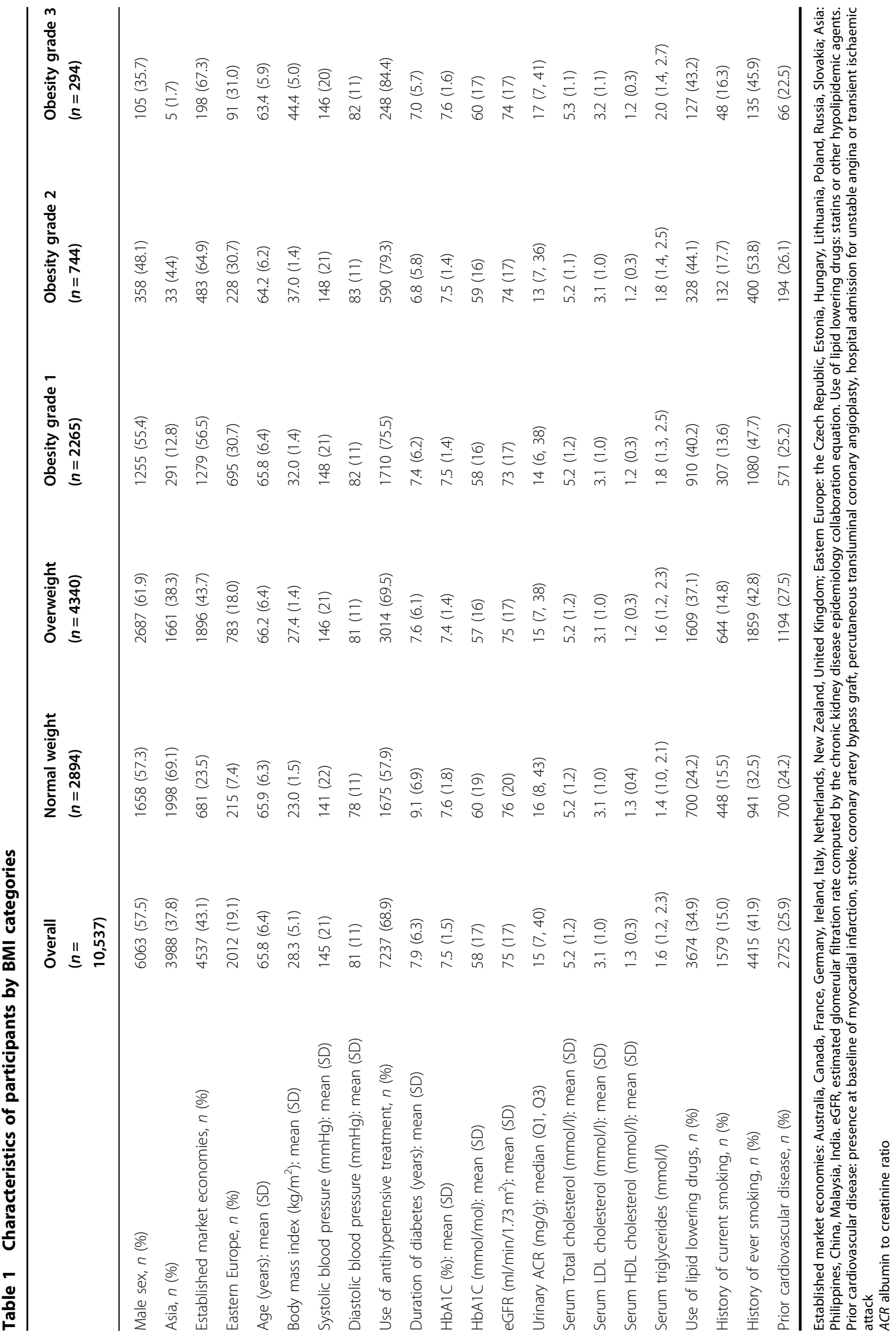



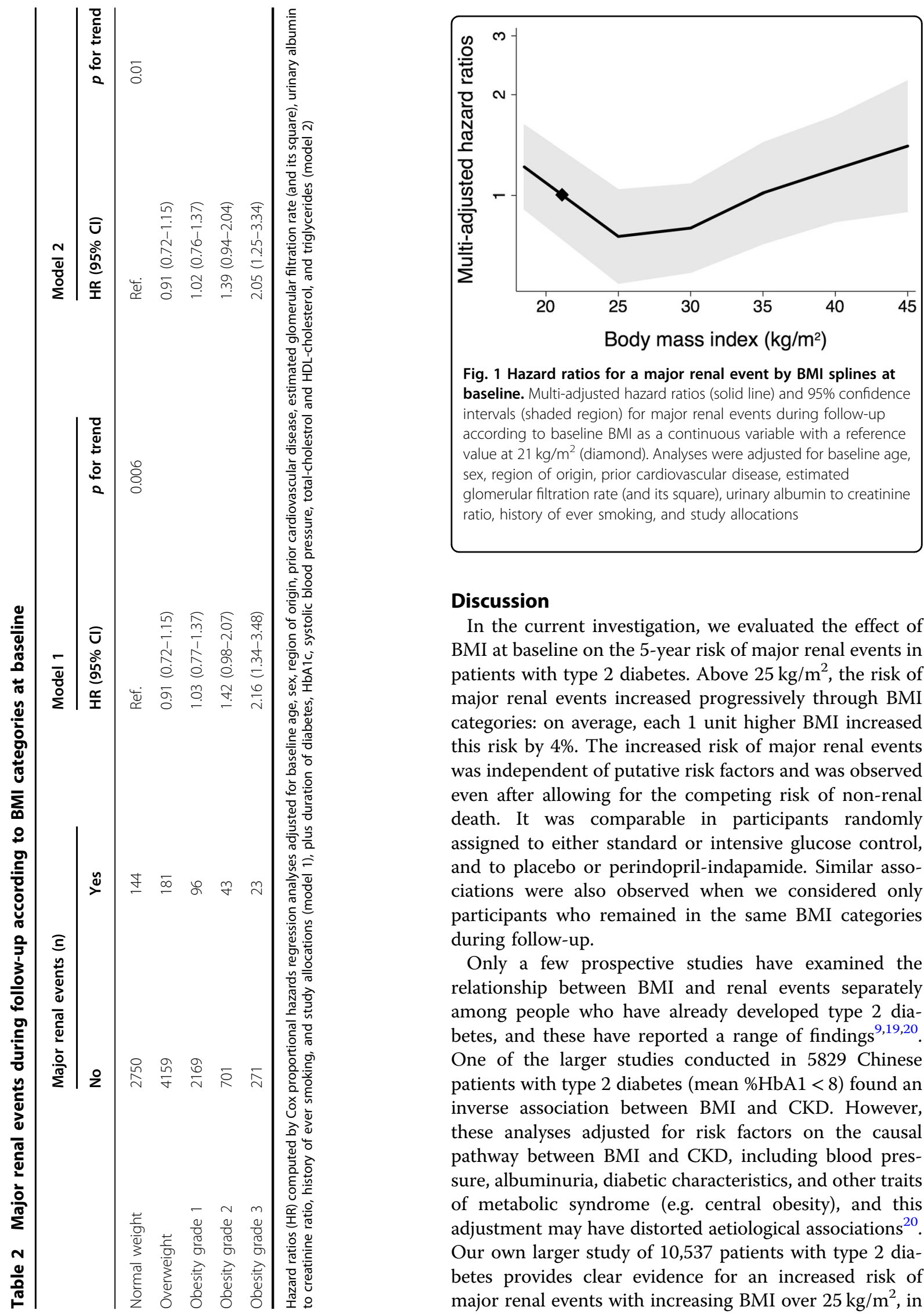

Fig. 1 Hazard ratios for a major renal event by BMI splines at baseline. Multi-adjusted hazard ratios (solid line) and 95\% confidence intervals (shaded region) for major renal events during follow-up according to baseline $\mathrm{BMI}$ as a continuous variable with a reference value at $21 \mathrm{~kg} / \mathrm{m}^{2}$ (diamond). Analyses were adjusted for baseline age, sex, region of origin, prior cardiovascular disease, estimated glomerular filtration rate (and its square), urinary albumin to creatinine ratio, history of ever smoking, and study allocations

\section{Discussion}

In the current investigation, we evaluated the effect of $\mathrm{BMI}$ at baseline on the 5 -year risk of major renal events in patients with type 2 diabetes. Above $25 \mathrm{~kg} / \mathrm{m}^{2}$, the risk of major renal events increased progressively through BMI categories: on average, each 1 unit higher BMI increased this risk by $4 \%$. The increased risk of major renal events was independent of putative risk factors and was observed even after allowing for the competing risk of non-renal death. It was comparable in participants randomly assigned to either standard or intensive glucose control, and to placebo or perindopril-indapamide. Similar associations were also observed when we considered only participants who remained in the same BMI categories during follow-up.

Only a few prospective studies have examined the relationship between BMI and renal events separately among people who have already developed type 2 diabetes, and these have reported a range of findings ${ }^{9,19,20}$. One of the larger studies conducted in 5829 Chinese patients with type 2 diabetes (mean $\% \mathrm{HbA} 1<8$ ) found an inverse association between BMI and CKD. However, these analyses adjusted for risk factors on the causal pathway between BMI and CKD, including blood pressure, albuminuria, diabetic characteristics, and other traits of metabolic syndrome (e.g. central obesity), and this adjustment may have distorted aetiological associations ${ }^{20}$. Our own larger study of 10,537 patients with type 2 diabetes provides clear evidence for an increased risk of major renal events with increasing BMI over $25 \mathrm{~kg} / \mathrm{m}^{2}$, in 


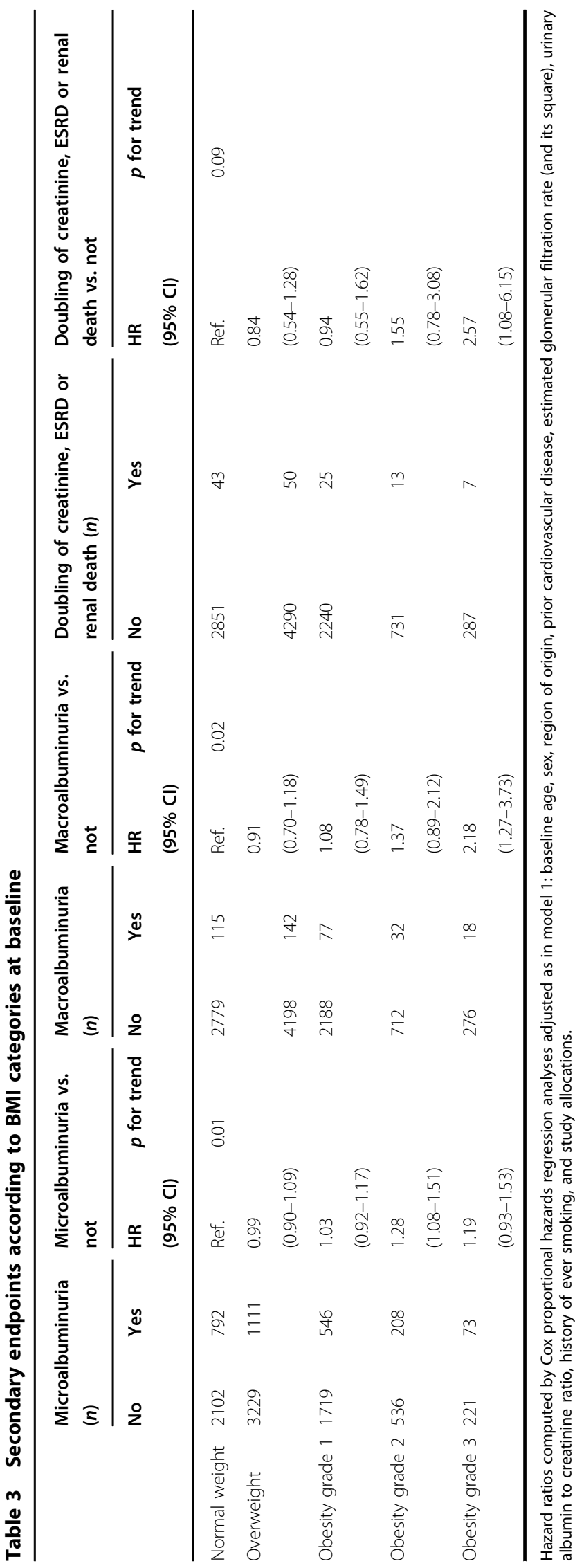

Cox models including BMI both as a categorical and as a continuous variable. The highest risk was observed in patients with morbid obesity.

Despite little apparent cross-sectional association between baseline BMI and baseline urinary ACR in our study, there was a clear positive association between BMI and development of new cases of microalbuminuria and macroalbuminuria, and these hazards were similar in size to the trend toward association between BMI and doubling of creatinine, ESRD or death. Furthermore, each additional unit of BMI over $25 \mathrm{~kg} / \mathrm{m}^{2}$ increased these endopints by 2 , 4 , and $5 \%$, respectively. A key mechanism for obesityassociated albuminuria is intraglomerular hypertension, which increases renal blood flow and fractional urinary albumin clearance ${ }^{21-24}$. The consequent mechanical stress results in glomerular enlargement (hypertrophy) and an increased distance between the neighbouring podocytes, damaging a key cellular layer of the glomerular filtration barrier $^{25}$ and perhaps causing podocyte death with focal segmental glomerulosclerosis ${ }^{26-28}$. Randomisation to perindopril + indapamide in ADVANCE reduced total renal events (major renal events plus new microalbuminuria) by $21 \%$ (relative risk $0.79,0.73-0.85)^{15}$. However, in our subgroup analyses, we found BMI-major renal events associations were not modified by allocated to perindopril + indpamide, which is consistent with a hypothesis that general adiposity may affect renal risk by mechanisms in addition to the haemodynamic stress of glomerular hyperfiltration.

Hyperglycaemia has been suggested as a metabolic podocyte stressor ${ }^{25}$. An inverse association between high insulin sensitivity (estimated by euglycemic clamp) and impaired renal function in a community-based cohort has been reported ${ }^{29}$, and pre-diabetes has been associated with directly measured evidence of hyperfiltration independent of $\mathrm{BMI}^{7}$. However, our subgroup analyses suggested that the BMI-major renal events association was not significantly modified by glycaemic control allocation (average HbA1c difference 0.7\%), despite the inverse relationship between $\mathrm{HbA1c}$ and weight ${ }^{30}$. Another mechanism by which adipose tissue may cause kidney disease is the visceral fat deposition in the renal sinus, which may compress the main renal artery and vein ${ }^{31-33}$, but measurements relevant to these mechanisms were not measured in this study.

Nevertheless, our findings are consistent with reports that weight loss may protect against the development of renal complications in overweight or obese individuals with type 2 diabetes. The Look AHEAD (Action for Health in Diabetes) trial showed that intensive lifestyle intervention, compared to standard education, resulted in $8 \%$ weight loss (on average $4 \mathrm{~kg}$ ) and a consequent $31 \%$ reduction (hazard ratio 0.69 [0.55-0.87]) in "very-high-risk CKD" (based in KDIGO risk charts) ${ }^{34}$. Weight loss may 


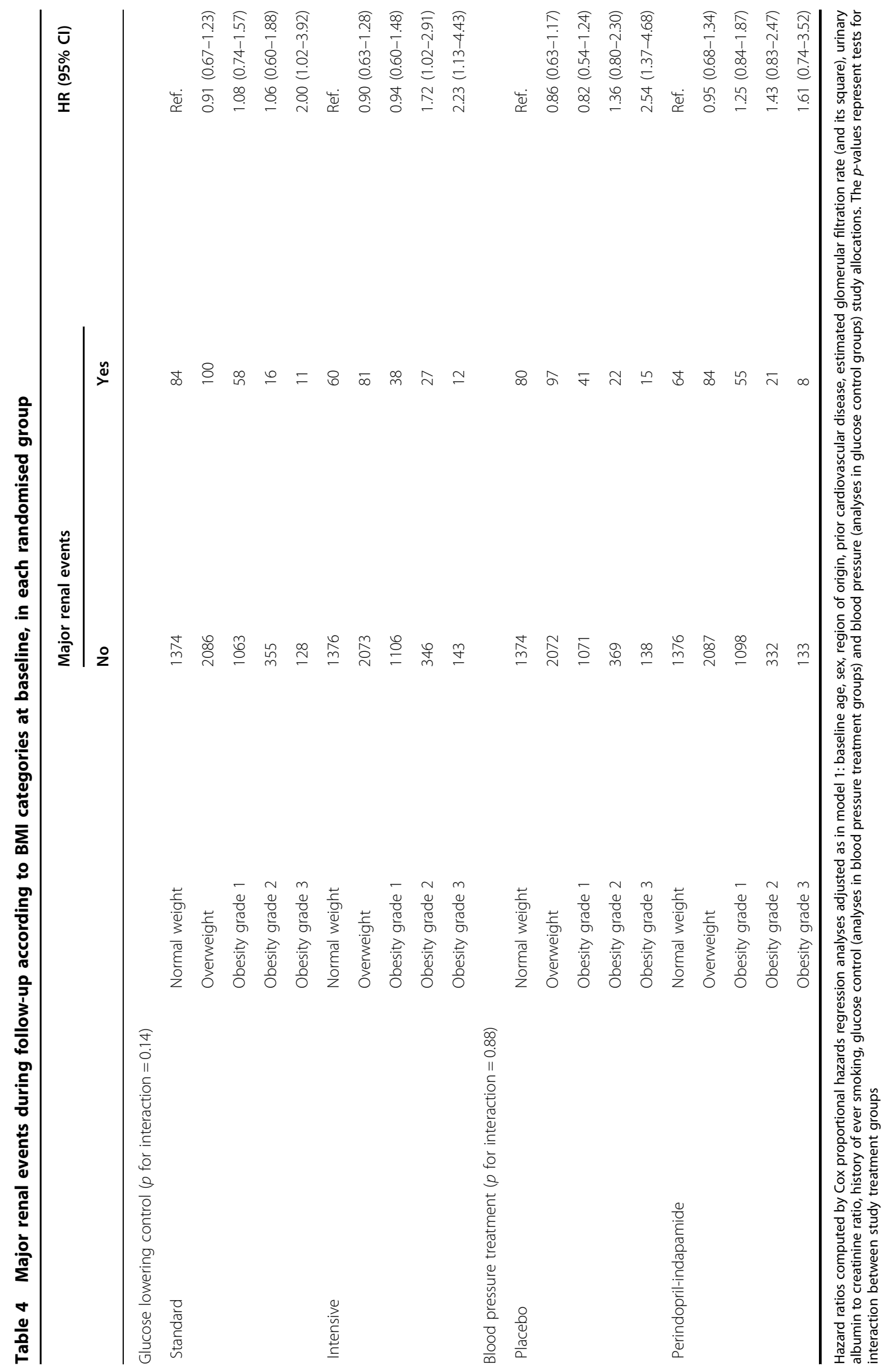


also be one of the mechanisms by which sodium-glucose co-transporter 2 inhibitors or analogues of glucagon-like peptide 1 reduce renal risk ${ }^{35-37}$. Lastly, bariatric surgery has been associated with an improvement in renal function $^{38-40}$. Regardless of the mechanism, higher risk with obesity suggests there may be greater absolute benefit from attention to all risk factors among patients with obesity and diabetes at risk for CKD progression.

The present investigation's key strength was its comprehensive clinical and biological characterisation of participants, and 5 years of prospective follow-up including pre-specified renal outcomes confirmed by an independent adjudication committee ${ }^{13}$. However, it is possible that the number of major renal events may have been insufficiently large to identify important differences between the randomised groups. Also, ADVANCE did not collect detailed data on body fat distribution, so that important differences between body-mass composition between participants could not be assessed for its relevance to major renal events. Furthermore, creatinine determinations were not isotope dilution mass spectrometry (IDMS) traceable in the ADVANCE trial as all participants were enroled before the international recommendations for IDMS alignment ${ }^{41,42}$.

In conclusion, obesity at different stages was an independent predictor of major renal events in patients with type 2 diabetes. Our findings encourage comprehensive and motivated weight loss programmes for improving the prevention of the development and progression of kidney complications in patients with both type 2 diabetes and obesity.

\section{Acknowledgements}

KM was supported by grants from the Société Francophone du Diabète (SFD) and the Association Diabète Risque Vasculaire (ADRV), and travel support from the Association pour le Développement de l'Enseignement et des Recherches auprès des universités, des centres de recherches et des entreprises d'Aquitaine (ADERA).

\section{Authors' contributions}

K.M., M.W., and J.Ch. designed the study; K.M. wrote the manuscript with assistance from J.Ch., W.H. and M.W.; L.Q. reviewed the statistical analyses. G.M., M.M., N.P., A.R., B.W., V.P. and J.Co. contributed to discussion and reviewed the manuscript. M.W. and K.M. are the guarantors of this work and, as such, had full access to all the data in the study and take responsibility for the integrity of the data and the accuracy of the data analysis. All authors approved the current version of the manuscript.

\section{Conflict of interest}

Dr. Kamel Mohammedi reports personal fees from Novo-Nordisk, outside the submitted work; Prof. John Chalmers reports grants and personal fees from Servier, outside the submitted work; Prof. Giuseppe Mancia reports personal fees from Boehringer Ingelheim, personal fees from CVRx, personal fees from Daiichi Sankyo, personal fees from Medtronic, personal fees from Merck \& Co. personal fees from Menarini, personal fees from Novartis, personal fees from Servier, outside the submitted work; Prof. Michel Marre reports grants and personal fees from Novo Nordisk, grants and personal fees from Sanofi, grants and personal fees from Eli Lilly, personal fees from Servier, grants and personal fees from Merck Sharp and Dohme, personal fees from Abbott, grants and personal fees from Novartis, personal fees from Astra Zeneca, outside the submitted work; Prof. Neil Poulter reports grants from the George Institute, grants from British Heart Foundation/Diabetes UKTThe George Institute, during the conduct of the study; grants from The George Institute, grants from BHF/ DUK/The George Institute, outside the submitted work; Prof. Bryan Williams reports personal fees from servier, outside the submitted work; and Prof. Mark Woodward reports personal fees from Amgen, outside the submitted work. No other potential conflict of interest relevant to this article was reported.

\section{Publisher's note}

Springer Nature remains neutral with regard to jurisdictional claims in published maps and institutional affiliations.

Supplementary Information accompanies this paper at https://doi.org/ 10.1038/s41387-017-0012-y.

Received: 6 January 2017 Accepted: 22 June 2017

Published online: 17 January 2018

\section{References}

1. NCD Risk Factor Collaboration. Worldwide trends in diabetes since 1980: a pooled analysis of 751 population-based studies with 4.4 million participants. Lancet 387, 1513-1530 (2016).

2. NCD Risk Factor Collaboration. Trends in adult body-mass index in 200 countries from 1975 to 2014: a pooled analysis of 1698 population-based measurement studies with 19.2 million participants. Lancet 387, 1377-1396 (2016).

3. Alberti, K. G. et al. Harmonizing the metabolic syndrome: a joint interim statement of the International Diabetes Federation Task Force on Epidemiology and Prevention; National Heart, Lung, and Blood Institute; American Heart Association; World Heart Federation; International Atherosclerosis Society; and International Association for the Study of Obesity. Circulation 120 (16), 1640-1645 (2009).

4. Collins, A. J. et al. US renal data system 2013 annual data report. Am. J. Kidney Dis. 63(1 Suppl.), A7 (2014).

5. Henegar, J. R., Bigler, S. A., Henegar, L. K., Tyagi, S. C. \& Hall, J. E. Functional and structural changes in the kidney in the early stages of obesity. J. Am. Soc. Nephrol. 12, 1211-1217 (2001).

6. Wang, Y., Chen, X., Song, Y., Caballero, B. \& Cheskin, L. J. Association between obesity and kidney disease: a systematic review and meta-analysis. Kidney Int. 73, 19-33 (2008).

7. Melsom, T. et al. Prediabetes and risk of glomerular hyperfiltration and albuminuria in the general nondiabetic population: a prospective cohort study. Am. J. Kidney Dis. 67, 841-850 (2016).

8. Fox, C. S. et al. Predictors of new-onset kidney disease in a community-based population. JAMA 291, 844-850 (2004).

9. Hsu, C. Y., McCulloch, C. E., Iribarren, C., Darbinian, J. \& Go, A. S. Body mass index and risk for end-stage renal disease. Ann. Intern. Med. 144, 21-28 (2006).

10. Iseki, K. et al. Body mass index and the risk of development of end-stage renal disease in a screened cohort. Kidney Int. 65, 1870-1876 (2004).

11. Lu, J. L. et al. Association of age and BMI with kidney function and mortality: a cohort study. Lancet Diabetes Endocrinol. 3, 704-714 (2015).

12. Vivante, A. et al. Body mass index in 1.2 million adolescents and risk for endstage renal disease. Arch. Intern. Med. 172, 1644-1650 (2012).

13. ADVANCE Management Committee. Study rationale and design of ADVANCE: action in diabetes and vascular disease--preterax and diamicron MR controlled evaluation. Diabetologia 44, 1118-1120 (2001).

14. Patel, A. et al. Intensive blood glucose control and vascular outcomes in patients with type 2 diabetes. N. Engl. J. Med. 358, 2560-2572 (2008).

15. Patel, A. et al. Effects of a fixed combination of perindopril and indapamide on macrovascular and microvascular outcomes in patients with type 2 diabetes mellitus (the ADVANCE trial): a randomised controlled trial. Lancet $\mathbf{3 7 0 ,}$ 829-840 (2007)

16. World Health Organization. Obesity. http://www.who.int/topics/obesity/en/ (2017).

17. KDIGO Group. KDIGO 2012 clinical practice guideline for the evaluation and management of chronic kidney disease. Kidney Int. 3(1 Suppl.), 1-150 (2013)..

18. Fine, J. P. \& Gray, R. J. A proportional hazards model for the subdistribution of a competing risk. J. Am. Stat. Assoc. 94, 496-509 (1999). 
19. Huang, W. H. et al. High body mass index reduces glomerular filtration rate decline in type II diabetes mellitus patients with stage 3 or 4 chronic kidney disease. Medicine 93(7), e41 (2014).

20. Luk, A. O. et al. Metabolic syndrome predicts new onset of chronic kidney disease in 5,829 patients with type 2 diabetes: a 5-year prospective analysis of the Hong Kong Diabetes Registry. Diabetes Care 31, 2357-2361 (2008).

21. Belhatem, N. et al. Impact of morbid obesity on the kidney function of patients with type 2 diabetes. Diabetes Res. Clin. Pract. 108, 143-149 (2015).

22. Chagnac, A. et al. Glomerular hemodynamics in severe obesity. Am. J. Physiol. Ren. Physiol. 278, F817-F822 (2000).

23. Chen, H. M. et al. Obesity-related glomerulopathy in China: a case series of 90 patients. Am. J. Kidney Dis. 52, 58-65 (2008).

24. D'Agati, V. D. et al. Obesity-related glomerulopathy: clinical and pathologic characteristics and pathogenesis. Nat. Rev. Nephrol. 12, 453-471 (2016).

25. Lewko, B. \& Stepinski, J. Hyperglycemia and mechanical stress: targeting the renal podocyte. J. Cell. Physiol. 221, 288-295 (2009).

26. Kambham, N., Markowitz, G. S., Valeri, A. M., Lin, J. \& D’Agati, V. D. Obesityrelated glomerulopathy: an emerging epidemic. Kidney Int. 59, 1498-1509 (2001).

27. Chen, H. M. et al. Podocyte lesions in patients with obesity-related glomerulopathy. Am. J. Kidney Dis. 48, 772-779 (2006).

28. Goumenos, D. S. et al. Early histological changes in the kidney of people with morbid obesity. Nephrol. Dial. Transplant. 24, 3732-3738 (2009).

29. Nerpin, E. et al. Insulin sensitivity measured with euglycemic clamp is independently associated with glomerular filtration rate in a community-based cohort. Diabetes Care 31, 1550-1555 (2008).

30. Mavian, A. A., Miller, S. \& Henry, R. R. Managing type 2 diabetes: balancing HbA1c and body weight. Postgrad. Med. 122, 106-117 (2010).
31. Schaffer, J. E. Lipotoxicity: when tissues overeat. Curr. Opin. Lipidol. 14, 281-287 (2003).

32. Montani, J. P. et al. Ectopic fat storage in heart, blood vessels and kidneys in the pathogenesis of cardiovascular diseases. Int. J. Obes. Relat. Metab. Disord. 28, S58-S65 (2004).

33. Foster, M. C. et al. Fatty kidney, hypertension, and chronic kidney disease: the Framingham Heart Study. Hypertension 58, 784-790 (2011).

34. The Look AHEAD Research Group. Effect of a long-term behavioural weight loss intervention on nephropathy in overweight or obese adults with type 2 diabetes: a secondary analysis of the Look AHEAD randomised clinical trial. Lancet Diabetes Endocrinol. 2, 801-809 (2014).

35. Wanner, C. et al. Empagliflozin and progression of kidney disease in type 2 diabetes. N. Engl. J. Med. 375, 323-334 (2016).

36. Marso S. P. et al. Semaglutide and cardiovascular outcomes in patients with type 2 diabetes. N. Engl. J. Med. 375, 1834-1844 (2016).

37. Marso, S. P. et al. Liraglutide and cardiovascular outcomes in type 2 diabetes. N. Engl. J. Med. 375, 311-322 (2016).

38. Chang, A. R. et al. Bariatric surgery is associated with improvement in kidney outcomes. Kidney Int. 90, 164-171 (2016).

39. Hou, C. C. et al. Improved renal function 12 months after bariatric surgery. Surg. Obes. Relat. Dis. 9, 202-206 (2013).

40. Navarro-Diaz, M. et al. Effect of drastic weight loss after bariatric surgery on renal parameters in extremely obese patients: long-term follow-up. J. Am. Soc. Nephrol. 17, S213-S217 (2006).

41. Myers, G. L. et al. Recommendations for improving serum creatinine measurement: a report from the Laboratory Working Group of the National Kidney Disease Education Program. Clin. Chem. 52, 5-18 (2006).

42. Mathew, T. H., Johnson, D. W. \& Jones, G. R. Chronic kidney disease and automatic reporting of estimated glomerular filtration rate: revised recommendations. Med. J. Aust. 187, 459-463 (2007) 